\title{
Capital social et usage des technologies de l'information et de la communication (TIC) : une analyse par les réseaux sociaux
}

\author{
par Alain Cucchi ${ }^{73}$ et Chantal Fuhrer ${ }^{74}$
}

\section{Résumé}

\begin{abstract}
Cet article traite des liens entre le capital social dans le réseau de travail et l'usage des TIC. II s'agit d'une recherche qui a pour objectif d'apporter des éléments de réponse sur les différents contextes d'usage des principaux médias de communication actuels et de montrer dans quelle mesure ils sont conditionnés par le capital social d'un individu dans un réseau de travail. Nous faisons l'hypothèse que les usages des différents outils de communication sont conditionnés par le capital social des individus. Pour traiter cette problématique, des données provenant de l'analyse des réseaux sociaux sont utilisées. Une étude exploratoire sur un groupe de 199 étudiants est menée. Les médias étudiés sont la messagerie, le téléphone, Skype et Facebook. Les résultats en cours sont présentés et analysés, avant de conclure sur des perspectives de recherches futures.
\end{abstract}

\section{Abstract}

This paper deals with social capital and ICT use.It is a research which aims to understand the context of ICT use of the main tools supporting relationships. We show in what extend does the social capital influence the use of those ICT tools. To do so, different measures from the Social Network Analysis are used. An exploratory study of 199 students is presented. Communication tools studied are electronic mail, telephone, Skype and FaceBook. Results in progress are presented then discussed. We conclude by future research perspectives.

Notre réseau relationnel a-t-il une valeur ? C'est la question que se propose de traiter la théorie du capital social. Elle considère que les relations tissées au sein d'un réseau social (physique et/ou virtuel) permettent de mobiliser des ressources. Dans le domaine des SI, la notion de relation se décline sous forme d'échanges d'informations. Les canaux de communication utilisés par les membres d'une communauté peuvent-ils traduire ces relations ? En d'autres termes, l'usage des technologies de l'information et de la communication (TIC) telles que la messagerie, le téléphone, la visio de type Skype/MSN et Facebook est-il influencé par le capital social des acteurs ? C'est la question que se propose de traiter cet article.

73. Alain Cucchi, Professeur des Universités, Université de la Réunion, IAE de La Réunion, CEMOI, alain.cucchi@univ-reunion.fr 74. Chantal Fuhrer, Maître de Conférences, Université de la Réunion, IAE de La Réunion, CEMOI, chantal.fuhrer@univ-reunion.fr 
S'intéresser à la communication dans les organisations revient à prendre en compte un phénomène multidimensionnel. Une étude sur tous les aspects de la communication n'existe pas. Un grand nombre d'études se focalisent sur certains de ses aspects. En Systèmes d'Information par exemple, la question de la relation entre la technologie et les usages a souvent été abordée (De Vaujany, 2000 ; Desanctis et Poole, 1994 ; Josserand, 2001 ; Kalika, 2000 ; 2002, p. 221236 ; Limayem et al, 1997 ; Rowe et Beal, 1998 ; Rowe, 2002 ; Yates et al, 1992 ; Bergeron et al, 1995 ; Boukef et Kalika, 2000 ; Crawford, 1982...). Des théories et modèles de recherche ont été élaborés afin de mieux appréhender cette question (Davis, 1989 ; Venkatesh et al., 2003 ; Fishbein et Ajzen, 1975 ; Ajzen, 1991 ; Rogers, 1995...). D'autres auteurs se sont également intéressés aux individus et aux types de relations tissées (Lasswell, 1973, p. 699). Ils peuvent alors se focaliser sur l'émetteur, sur le récepteur ou sur les deux (Watzlawick et al., 1967 ; Moreno 1970 ; Short et al, 1976). Par conséquent, chaque étude sur la communication privilégie certains aspects, plutôt que d'autres.

A l'heure actuelle, les connaissances relatives aux interactions usage des TIC et capital social sont insuffisantes (Yang et al. 2009, p.184). Si les recherches sur les usages des TIC sont nombreuses, plus rares sont celles qui prennent en considération à la fois le réseau d'échange et la position de l'acteur dans ce dernier. Les approches empruntées à la sociologie, telles que l'analyse des réseaux sociaux et l'analyse du capital social s'attachent à combler cette lacune. Notre recherche se positionne dans cette perspective.

Cet article traite des liens entre le capital social dans le réseau de travail et l'usage des TIC. II s'agit d'une recherche en cours, qui a pour objectif d'apporter des éléments de réponse sur les différents contextes d'usage des principaux médias de communication actuels et de montrer dans quelle mesure le capital social d'un individu dans un contexte de travail conditionne l'usage des médias électroniques. Nous faisons l'hypothèse que le capital social d'un individu influence l'usage des différents médias de communication électronique.

L'article s'articule autour de grandes parties. Les deux premières présentent les théories mobilisées pour concevoir notre modèle de recherche, concernant les usages des TIC et la notion de capital social. Le modèle de recherche est alors présenté dans la troisième partie. Dans une quatrième partie, la méthodologie utilisée est décrite, ainsi que les traitements effectués. Les résultats sont présentés dans la cinquième partie puis discutés dans une dernière partie avant la conclusion. 


\section{Les usages des TIC}

Plusieurs travaux cherchent à identifier les facteurs techniques, économiques, sociologiques, psychologiques..., qui influencent l'usage des médias électroniques. Si nous déclinons les principales théories en précisant les variables qu'elles utilisent (variables dépendantes et variables indépendantes), elles tiennent peu compte des aspects relationnels associés au réseau de communication. Dans la Théorie Unifiée de l'Acceptation et de l'Utilisation de la Technologie (UTAUT), l'acceptation et l'usage de la technologie est expliquée par plusieurs variables : la performance attendue, l'effort attendu, l'influence sociale, les conditions facilitatrices, le genre, l'âge, l'expérience et la volonté d'usage (Venkatesh et al., 2003, p. 447). Dans ce modèle, l'influence sociale représente le degré de perception d'un individu selon lequel d'autres personnes importantes à ses yeux estiment qu'il devrait utiliser le nouveau système. L'aspect relationnel est ici traduit par le regard des autres sur soi. Dans la théorie de l'action raisonnée (TRA) (Fishbein et Ajzen, 1975) le comportement d'un individu est conditionné par son intention d'accomplir telle ou telle action, laquelle est déterminée par l'attitude de la personne envers l'action et par la " norme subjective ». La norme subjective représente l'idée que la plupart des personnes importantes aux yeux de l'individu pensent que l'individu devrait (ou ne devrait pas) accomplir l'action. L'aspect relationnel est également traduit ici par le regard des autres sur le comportement individuel. De même, dans la Théorie du Comportement Plannifié (PBT) (Ajzen, 1991), l'idée de norme subjective est reprise, correspondant aux opinions perçues des autres. L'individu est motivé à se conformer aux attentes des autres. Si ces recherches font référence aux aspects relationnels (Rai et al., 2009), elles ne traitent pas de façon explicite la notion de capital social (Yang et al. 2009, p.191). De plus, ces dernières intègrent pour la plupart les usages des outils de manière non différenciée. Or dans les faits, les usages se superposent, tel que nous le montrent Kalika et al. (2005) avec l'effet « millefeuille ».

II en est de même pour les théories et auteurs plus proches de la sociologie. Ces derniers présentent des modèles où il existe des relations réciproques et récursives entre le média et la communication, se basant sur des théories telles que la structuration (Giddens, 1988), les genres (Contractor et Eisenberg, 1990 ; DeSanctis et Poole, 1994 ; Yates et Orlikowski, 1992, 2002...). Dans le cas de la théorie de la structuration, l'accent est mis sur l'émergence d'un système de règles qui contraint et facilite les interactions sociales. Dans celui de la théorie des genres, l'attention porte sur le développement de " normes » sociales de communication (contenu des communications) pour structurer les interactions.

Cependant, ces théories n'intègrent pas les conditions relationnelles liées aux usages. Ce problème se révèle avec d'autant plus d'acuité dans les technologies de la communication. Cette recherche vise à combler cette lacune. Elle 
propose donc l'intégration des relations sociales dans la mesure des usages. La problématique ici se pose en termes de structuration du réseau d'échanges, favorisant l'usage d'un média. Les travaux se basent sur l'analyse des réseaux sociaux.

\subsection{L'analyse des réseaux sociaux}

L'analyse des réseaux sociaux (Social Network Analysis ou SNA) est une approche qui considère la société comme un système d'acteurs - individus, groupes, organisations - liés par un certain nombre de relations. Ces relations peuvent être de plusieurs natures et l'analyse consiste à étudier la présence (ou l'absence) de ces relations (Tichy, 1981 ; Brass and Burkart, 1992). Un réseau social correspond à un ensemble de nœuds (individus, organisations) reliés par un ensemble de relations sociales (amitiés, transfert de fonds...) (Laumann et Pappi, 1976 ; Lazega, 1998). L'analyse du réseau inclut la description de la structure et de la configuration des relations, ainsi que l'identification de leurs causes et de leurs conséquences (Tichy, 1981; Laumann and Pappi, 1976 ; Nohria, 1992). De ce point de vue, les acteurs sociaux interagissent. Cette perspective consiste à se focaliser sur ces interactions (entre des individus au sein d'un groupe, entre des groupes au sein d'une organisation, etc.) et permet une meilleure description et compréhension des activités de l'organisation (Lamb and Kling, 2003). Ainsi, cette perspective permet de prendre en compte à la fois deux types de paramètres : ceux correspondant à l'acteur social (relativement aux autres acteurs du réseau) et ceux correspondant à la relation qui relie chaque acteur au réseau global. Parmi les nombreux concepts manipulés par le SNA (Social Network Analysis), celui sans doute le plus étudié est la centralité.

\subsection{La centralité des acteurs}

La conception usuelle de la centralité requiert l'étude de l'implication de chaque acteur dans un réseau. Un système est dit centralisé quand toutes les relations concernent un unique acteur (Burt, 1980, p.117 ; 1982, p.33). Ceci contribue fortement à la description de l'inégalité dans l'implication de chacun dans un réseau de relations (Burt, 1980, p.117). Bien que certains auteurs soulignent l'importance des positions périphériques (Granovetter, 1973), on suppose généralement que la centralité dans un réseau traduit « l'importance » de l'individu dans son réseau. Des auteurs ont lié cette idée de centralité à celle de pouvoir (Bonacich, 1987), d'influence ou de leadership (Wasserman and Faust, 1994). Les individus les plus centraux dans un réseau occupent ainsi des relations privilégiées dans les échanges.

Comment identifier les acteurs « les plus importants » dans un réseau ? Plusieurs auteurs s'accordent à dire que les acteurs les plus importants sont généralement localisés dans des emplacements stratégiques dans le réseau. Si on définit la 
centralité d'un graphe comme son centre, est-ce que celle-ci contient réellement la personne la plus importante ? Un acteur est important si « les liens concernant cet acteur le rend particulièrement visible des autres acteurs du réseau " (Wasserman and Faust, 1994, p.172). Ces liens ne sont pas uniquement des liens directs mais incluent ceux indirects, faisant intervenir des liens intermédiaires.

Des auteurs tels que Knoke et Burt ont distingué deux classes d'importance : la centralité et le prestige (Wasserman and Faust, 1994, p.173). La définition la plus intuitive de la centralité consiste à considérer qu'un acteur est central s'il est impliqué dans plusieurs relations (Wasserman and Faust, 1994). Plusieurs compléments etévolutions de ce concept ontété proposés depuis (voir Wasserman and Faust, 1994 p. 174). De même, plusieurs mesures ont été proposées par les auteurs afin d'évaluer "l'importance » d'un acteur. Les indices diffèrent en fonction du niveau d'analyse. Ce dernier peut être un acteur (on fera référence à l'activité (degré...), la position (proximité, position intermédiaire)), une dyade (les mesures dyadiques), ou le réseau dans son ensemble ("vecteur propre ", " position intermédiaire globale »). Une autre différence se situe au niveau des données. Les relations peuvent être symétriques (les relations de $A$ vers $B$ sont égales à celles de $B$ vers $A$ ) ou asymétriques (cas inverse). Dans le cas où les relations de communication sont symétriques, les indices concernés sont appelés " centralités ». A l'inverse, dans un cas d'asymétrie du flux de communication, les indices concernés sont appelés « influence ». Une description détaillée des principaux indices de centralité est présentée dans Wasserman and Faust (1994). Les mesures de centralité de Freeman (1979) figurent parmi les plus célèbres. Ce dernier propose trois mesures de la centralité : le degré (degree), la proximité (closeness) et la position intermédiaire (betweeness). Le degré correspond au nombre de connexions directes qu'un individu a vis-à-vis des autres membres de son réseau. Cette mesure considère qu'un individu est d'autant plus central qu'il est directement lié à un grand nombre d'individus du réseau. Cette notion de degré se décline en deux parties : les échanges que reçoit l'individu de la part des membres du réseau (le In-Degree) et les échanges que l'individu émet pour les membres du réseau (le Out-Degree). La proximité fournit un point de vue plus global de la centralité puisqu'elle considère la proximité d'un individu vis-à-vis des autres. Enfin, la position intermédiaire considère qu'un individu peut bien être faiblement connecté aux autres, voire éloigné, mais servir d'intermédiaire dans les échanges entre les autres individus du réseau.

D'autres mesures de centralité peuvent être utilisées permettant de mesurer les multiples aspects de l'importance d'un individu, conférant à l'individu central plus d'informations, de pouvoir, de prestige, ou d'influence. Celles que nous avons utilisées dans notre recherche sont résumées dans le tableau ci-dessous. 


\begin{tabular}{|c|l|}
\hline \multirow{2}{*}{$\begin{array}{c}\text { Degré (degree) } \\
\text { In-degree }\end{array}$} & $\begin{array}{l}\text { Une mesure de l'activité. Les acteurs centraux ont plus de relations que } \\
\text { les autres acteurs du réseau. }\end{array}$ \\
\cline { 2 - 2 } $\begin{array}{c}\text { Out-degree } \\
\text { Vecteur propre } \\
\text { (Eigenvector) }\end{array}$ & \begin{tabular}{l} 
Connexions orientées des membres du réseau vers l'individu \\
\cline { 2 - 2 } de la centralité des acteurs avec lesquels il est connecté.
\end{tabular} \\
\hline Authority & $\begin{array}{l}\text { Pour un graphe valué, on peut ajouter tous les poids des relations en } \\
\text { partance et tous les poids des relations à destination d'un individu }\end{array}$ \\
\hline Hub & Nœud ayant un degré et une position intermédiaire importants. \\
\hline
\end{tabular}

Tableau 1 : Mesures de la centralité utilisées.

Ces mesures nous permettent de mieux interpréter la notion de centralisation d'une structure inhérente à un réseau de communication. Cette structure est influencée par le capital social de l'individu.

\section{Le concept de capital social}

Au cours des 10 dernières années, le concept de capital social a été mobilisé dans de nombreux travaux et dans des disciplines différentes. Au début des années 1990, le nombre de travaux mobilisant ce concept n'excédait pas 3. En 2003, plus de 300 peuvent être dénombrés (Putnam, 2004). Cette profusion engendre une hétérogénéité des définitions, des interprétations et des approches. La question de la définition du concept de « capital social » est en conséquence difficile, ambiguë et mobilise plusieurs sources d'interprétation. Sans chercher l'exhaustivité, les paragraphes suivants s'attachent à décrire les éléments communs aux différentes approches en indiquant au préalable quelques points de repère.

\subsection{Quelques points de repère}

Dans son ouvrage Bowling alone (2000), Putnam considère que le concept de « capital social » a été évoqué à l'origine par Hanifan dans les années 1920. Pour ce dernier, le capital social recouvre la bienveillance, l'amitié, la sympathie et les rapports sociaux entre les membres d'une même unité sociale. De son point de vue, chaque individu est en relation avec les autres, ce qui permet l'accumulation de capital social. Le capital social est donc un phénomène supporté par le réseau des relations individuelles, à caractère cumulatif par le biais des interactions sociales. Ses effets sont bénéfiques à un niveau individuel et collectif.

Dans son étude du concept de classe, Bourdieu a identifié trois aspects du concept de capital : économique (revenus, patrimoine), culturel (langage, savoir et savoirfaire, capital intellectuel...) et le capital social. Une des caractéristiques de ces 
ressources est de trouver leur efficacité dans leur environnement social. Bourdieu $(1980,1985)$ définit le capital social comme «l'ensemble des ressources actuelles ou potentielles qui sont liées à la possession d'un réseau durable de relations plus ou moins institutionnalisées d'interconnaissance et d'interreconnaissance ; ou, en d'autres termes, à l'appartenance à un groupe, comme ensemble d'agents qui ne sont pas seulement dotés de propriétés communes... mais sont aussi unis par des liaisons permanentes et utiles ». Ainsi, le volume de capital social d'un individu dépend de la densité, de la nature et de l'étendue du réseau de ses relations mais également du volume de capital de ceux auquel il est relié. Bourdieu utilise ce concept dans son interprétation du pouvoir, ces relations sociales qui augmentent la capacité d'un acteur à développer ses propres intérêts.

Coleman (1988) introduit le concept de capital social en s'appuyant au départ sur deux conceptions pour décrire et expliquer l'action sociale. La première étant celle des sociologues, qui considèrent que l'acteur est sociabilisé et que l'action est gouvernée par des normes sociales, des règles et des obligations. La deuxième étant celle des économistes, qui considèrent que l'acteur a des objectifs qu'il se fixe de manière indépendante, qu'il agit de façon indépendante et qu'il est entièrement guidé par des intérêts personnels (p.95). En combinant ces deux approches, il introduit la notion de capital social (p.96). II emprunte aux économistes le principe de l'action rationnelle pour l'utiliser dans l'analyse des propriétés des systèmes sociaux (p.97). La notion de capital social y est présentée parallèlement aux concepts de capital financier, de capital physique et de capital humain, mais exprimée à travers des relations entre les personnes (p.118). Il décline la notion de capital social selon trois aspects : les obligations et attentes, qui dépendent de la relation de confiance (trustworthyness) qui existe dans l'entourage social, les capacités de flux informationnel de la structure sociale ainsi que les normes accompagnées de sanctions. Coleman (1988) développe ainsi une réflexion sur le capital social centrée sur les fonctions. Comme d'autres formes de capital, le capital social est productif, rendant possible la réalisation de certaines fins qui n'auraient pu être atteintes sans lui.

Le concept de " capital social » de Putnam $(1993,1995)$ s'appuie sur trois éléments : les obligations morales et les normes, les valeurs sociales (en particulier la confiance) et les réseaux sociaux (notamment les associations). Selon lui, le capital social se réfère à des caractéristiques des organisations sociales telles que les réseaux, les normes et la confiance. II facilite la coordination et la coopération, développant ainsi des bénéfices mutuels. Le capital social est un facteur qui influence positivement la performance économique et l'intégration sociale. Cet auteur pose la confiance comme le concept central permettant de développer le capital social. Elle émerge d'interactions locales et d'attentions réciproques. Elle permet le développement de " bonnes actions » qui seront récompensées par le développement positif de relations communes. Elle est nécessaire dans des environnements complexes, ambigus, contingents et risqués où les acteurs 
ont des difficultés à anticiper les réactions des partenaires. Ainsi, le concept de capital social de Putnam peut être interprété comme un mécanisme qui renforce le consensus et l'intégration des valeurs (sociologie de l'intégration) alors que celui de Bourdieu peut être interprété comme un mécanisme impliqué dans les conflits sociaux et dans les structures du pouvoir (sociologie du conflit) (Martti Siisiäinen, 2000).

Burt (1992) considère le capital social d'un point de vue relationnel plutôt que centré sur les attributs individuels. Le capital social se rapproche du capital relationnel c'est-à-dire les amis, les collègues, les partenaires... D'un point de vue individuel, l'utilité du capital social dépend des " trous structuraux ». II faut comprendre par cette expression la séparation entre deux contacts non redondants. Des contacts seront considérés comme redondants s'ils se connaissent directement ou indirectement : ils seront alors en situation " d'équivalence structurale ». Autrement dit, l'utilité du capital social augmente si les membres du réseau relationnel ne se connaissent pas. Cette non redondance des contacts permet à l'acteur de diversifier les sources d'information et de mettre en œuvre des formes de contrôle dans les échanges. Selon Burt, la propriété et les actifs humains constituent les capacités de production de la firme. Les relations intra et interfirmes constituent le capital social. Le capital social représente d'une part la structure des contacts au sein d'un réseau et d'autre part les ressources qu'ils détiennent. Dans le premier cas on décrit comment on atteint et dans le deuxième cas on décrit qui on atteint (Burt, 1992, p.61).

En synthèse, les promoteurs du concept de capital social considèrent que les relations sociales, s'appuyant sur des normes, des valeurs partagées et la confiance, facilitent la coordination ou la coopération entre les individus. II s'agit d'une ressource bénéficiant à un niveau collectif et individuel. Les définitions du capital social sont multiples et diffèrent selon le niveau d'analyse individuel ou collectif (Yang et al. 2009, p.186). Dans le cas où le capital est considéré en variable dépendante, il s'agit, dans le domaine des SI, d'étudier les impacts des TIC sur le capital social. En tant que variable indépendante, il s'agit d'étudier les effets du capital social sur l'utilisation et le développement des TIC. Ces auteurs montrent qu'il y a relativement peu de recherche sur le thème du capital social en tant que variable indépendante (p.190). Ce travail s'inscrit dans cette dernière perspective et vise à compléter la recherche actuelle dans ce domaine.

Les relations, les interactions sociales sont donc au cœur du concept de capital social. C'est également le cas pour le réseau social.

\subsection{Capital social et réseau social}

Le capital social trouve son origine dans les interactions sociales entre les acteurs appartenant à un groupe identifié. Cette notion de groupe d'acteurs est également 
étudiée dans le domaine des réseaux sociaux. Ces deux concepts, capital social et réseau social sont très liés. Le sociologue Bourdieu, cité par Montes et Pronovost (2007) considère le capital social comme la somme des ressources, pour un individu ou un groupe, émanant d'un réseau durable de relations plus ou moins institutionnalisées, de contacts mutuels et de reconnaissance (p.7). Adler et Kwon stipulent que le capital social « constitue la bienveillance disponible aux individus et aux groupes. Son origine provient de la structure et le contenu des relations sociales des acteurs. Son effet se manifeste sous forme d'information, d'influence et de solidarité disponible à l'acteur (p.8). Selon Lin, le capital social est constitué « des ressources encastrées dans un réseau social, [...] d'ailleurs le capital social représente les actifs disponibles dans le réseau » (p.8). Le concept de réseau est donc très proche.

L'analyse des réseaux sociaux (Social Network Analysis ou SNA) est une approche qui considère la société comme un système d'acteurs - individus, groupes, organisations - liés par un certain nombre de relations. Ces relations peuvent être de plusieurs natures et l'analyse consiste à étudier la présence (ou l'absence) de ces relations (Tichy, 1981 ; Brass and Burkart, 1992). Un réseau social correspond à un ensemble de nœuds (individus, organisations) reliés par un ensemble de relations sociales (amitiés, transfert de fonds...) (Laumann et al., 1978). L'analyse du réseau inclut la description de la structure et de la configuration des relations, ainsi que l'identification de leurs causes et de leurs conséquences (Tichy, 1981 ; Laumann and Pappi, 1976 ; Nohria, 1992). De ce point de vue, les acteurs sociaux interagissent. Cette perspective consiste à se focaliser sur ces interactions (entre des individus au sein d'un groupe, entre des groupes au sein d'une organisation, etc.) et permet une meilleure description et compréhension des activités de l'organisation (Lamb and Kling, 2003). Ainsi, cette perspective permet de prendre en compte à la fois deux types de paramètres : ceux correspondant à l'acteur social (relativement aux autres acteurs du réseau) et ceux correspondant à la relation qui relie chaque acteur au réseau global.

Les auteurs qui se sont intéressés aux deux concepts tiennent tous pour acquis que les deux notions de capital social et de réseau social sont enchevêtrées (Montes et Pronovost, 2007, p.9). Certains comme Putman considèrent le capital social comme une qualité des groupes (régit par la loi, confiance) et d'autres comme Burt le considère comme la valeur qu'un individu peut tirer de ses relations sociales (Burt, 1980, 1982). Freeman propose une association entre les différentes dimensions du capital social (confiance, fidélité, vision partagée...) et l'utilisation des outils de topographie (sociomatrice des interactions formelles et informelles) sur le réseau (Montes et Pronovost, 2007, p.10). L'idée de l'utilisation simultanée des concepts de capital social et de réseau semble donc incontournable. C'est le cas de cette recherche. Pour ce faire, nous nous attachons maintenant à retenir des mesures de ce concept de capital social. 


\subsection{Les mesures du capital social}

Ce concept de capital social est utilisé par certaines recherches en gestion et en Systèmes d'Information en particulier (Cucchi et al. 2009 ; Yang et al., 2009). En gestion, le capital social intervient essentiellement dans le domaine de la gestion des connaissances.

Nous pouvons retenir la définition de Nahapiet et Ghoshal $(1997,1998)$ qui considèrent le capital social comme "la somme des ressources potentielles et réelles encastrées dans, disponibles au travers de, et venant du réseau des relations possédées par un individu ou une unité sociale ». Ainsi, ces auteurs adoptent une perspective élargie en intégrant à la fois la dimension structurelle du réseau et les ressources potentiellement mobilisables par le réseau.

Comme le fait remarquer Putnam (1995, 2004), le concept même de capital social est encore en construction. L'apport principal de Nahapiet et Ghoshal (1997) est de répondre à un besoin de clarification et d'opérationnalisation du concept de capital social. Trois attributs du capital social sont considérés : le structurel, le cognitif et le relationnel.

\section{- Le structurel}

La dimension structurelle fait référence au modèle de connexions entre les acteurs, incluant les liens à travers le réseau, la configuration du réseau en matière de densité, de connectivité etc. La dimension structurelle " concerne les propriétés du système social et du réseau de relations comme un tout. $\mathrm{Ce}$ terme décrit les configurations impersonnelles de liens entre les personnes ou les unités » (p. 244). Pour reprendre Burt (1992), la dimension structurelle décrit l'ensemble des connexions entre acteurs, la présence ou l'absence de liens entre acteurs en répondant aux questions : qui pouvez-vous atteindre ? Comment pouvez vous les atteindre ? La structure du réseau ne prédit pas directement les attitudes ou les comportements, elle prédit des similarités entre les attitudes et les comportements. (Burt, 1992, p.60). Dès 1982, il propose une analyse de la structure du réseau à travers six modèles, selon qu'il s'agisse d'une approche relationnelle ou d'une approche positionnelle (Burt, 1982, p.30). La plupart de ces modèles mesurent l'intégration sociale. Un acteur est « isolé » à la périphérie du système s'il n'a pas de relations avec les autres du système. Deux modèles ont été utilisés pour décrire l'intégration sociale des acteurs : la centralité et le prestige. Un acteur a une position centrale dans la mesure où il est impliqué dans toutes les relations dans le réseau (Burt, 1982, p.33).

D'autres auteurs proposent dans ce même registre d'étudier les caractéristiques structurelles, mais également les caractéristiques des liens et les caractéristiques des participants clé à l'intérieur du réseau. Tichy (1981), dans son chapitre sur l'analyse des réseaux sociaux, propose d'étudier les caractéristiques des liens 
selon quatre axes : la réciprocité, la clarté des normes, l'intensité, la multiplexité (multiplexity). II propose également d'étudier les caractéristiques des participants clés, selon qu'on les considère comme étoile (star), comme agent de liaison, comme pont (bridge), comme gardien des relations ou comme acteur isolé (Tichy, 1981, p.229).

Degenne et Forsé soulignent l'importance de la structure comme contrainte et effet émergent : La structure est appréhendée concrètement comme un réseau de relations, mais elle est aussi " contrainte ». Elle est le réseau en tant que contrainte qui pèse sur les choix, les orientations, les comportements, les opinions... des individus. L'analyse de réseau est donc le moyen d'une analyse structurale dont le but est de montrer en quoi la forme du réseau est explicative des phénomènes analysés (Degenne et Forsé, 1994, p.8). Par conséquent, nous conviendrons avec Nohria que les réseaux sont plus des processus que des structures, étant continuellement formés et reformés par les actions des acteurs qui en retour sont contraints par les positions structurelles dans lesquelles ils se trouvent (Nohria, 1992, p.7).

Cette dimension structurelle permet de caractériser deux niveaux d'analyse. Au niveau individuel, elle permet de mesurer un ensemble d'indicateurs sur la position de l'acteur dans le réseau : centralité, prestige... (cf. Wasserman, Faust, 1994). Ces mesures permettent alors d'évaluer l'intégration sociale de l'acteur dans le réseau (Burt, 1982).

Une mesure de la dimension structurelle proposée par Nahapiet et Ghoshal (1997) comprend les liens dans le réseau (qui connais-tu ?), la configuration du réseau et l'organisation appropriée.

En ce qui concerne cette recherche, la dimension structurelle sera traitée de deux façons :

- Avec qui travailles-tu?

Étant donné que nous étudions les relations dans un contexte de travail, nous considérons dans cette recherche les liens dans le réseau permettant de savoir avec qui l'individu travaille.

\section{- L'utilisation de l'analyse des réseaux sociaux}

En fait, nous considérons qu'il n'existe pas une structure unique mais plusieurs structures. Les caractéristiques homogènes des structures dépendent du contexte d'usage. Nous utilisons l'analyse des réseaux sociaux pour comparer les structures de relations.

Ainsi, dans la dimension structurelle, nous nous focalisons sur la centralité de l'individu dans son réseau de travail et nous pensons que cette centralité influence positivement les usages des TIC. 


\section{- Le cognitif}

Pour que la communication entre acteurs ait du sens, cela nécessite de partager une connaissance du contexte. Cette connaissance commune (" common knowledge ") constitue la dimension cognitive. Elle fait référence aux ressources qui permettent une compréhension commune, des représentations et des systèmes de signification communs, partageant un langage commun, des codes et des histoires. Une mesure de la dimension cognitive proposée par Nahapiet et Ghoshal, (1997) comprend 3 aspects :

- les codes et le langage partagés

Le langage a une fonction sociale importante dans les relations sociales. C'est le moyen par lequel les personnes échangent des informations et conduisent leurs affaires. En partageant un langage, cela permet aux acteurs de se rencontrer plus facilement et d'évaluer les bénéfices potentiels dans l'échange.

\section{- les histoires partagées}

Les histoires, mythes et métaphores fournissent également aux groupes des moyens puissants pour échanger et préserver des systèmes riches de significations. Les métaphores, en s'affranchissant des contextes, permettent la combinaison de capacités d'imagination, d'observation et de cognition. Les histoires facilitent le partage de connaissances tacites et améliorent les pratiques.

- la connaissance tacite partagée

Partant du fait que nous savons plus que nous ne pouvons le dire, Polanyi (1967) supporte l'idée que toute forme de connaissance présente des aspects théoriques et pratiques où la dimension tacite est essentielle.

En ce qui concerne ce travail, nous considérons cette dimension cognitive dans le choix du bon comportement individuel au travail. Ceci nous permet d'interpréter le niveau de proximité, de similarité dans leur vision et de positionner les acteurs fédérateurs. Par conséquent, l'idée de vision partagée représente la dimension cognitive et celle-ci doit influencer positivement l'usage des TIC.

\section{- La dimension relationnelle}

La dimension relationnelle fait référence aux actifs mobilisés à travers les relations, incluant les attributs tels que la confiance, les normes et les sanctions. Une mesure de la dimension relationnelle proposée par Nahapiet et Ghoshal, (1997) comprend 4 aspects :

- la confiance

La confiance est une des pierres angulaires de la dimension relationnelle. Les acteurs développant un sentiment de confiance s'engagent plus dans des échanges sociaux. Ils ont tendance à prendre plus de risques dans les échanges, 
à expérimenter plus de choses. Enfin, la confiance permet de s'adapter plus facilement à des situations complexes et changeantes, en s'affranchissant plus ou moins des risques de comportements opportunistes.

\section{- les normes}

Les normes de coopération facilitent l'ouverture pour des échanges de connaissances. Des normes d'ouverture à la critique permettent également d'éviter les effets de groupe qui inhibent le développement de capital intellectuel.

- les obligations et attentes

Les obligations et attentes constituent un engagement ou un devoir pour la réalisation d'activités dans le futur. Elles agissent comme des " crédits d'erreur " devant être remboursés par des comportements futurs.

\section{- l'identification}

C'est le processus par lequel les individus se reconnaissent au travers des valeurs d'un groupe ou comme appartenant à un groupe. L'identification à un groupe renforce les processus collectifs et les possibilités d'échanges. Elle agit comme une ressource influençant la motivation à échanger.

Cette dimension relationnelle est ainsi multiforme. Elle a des liens étroits avec la fidélité, l'engagement, l'implication. Elle peut également représenter les relations d'autorité ou de pouvoir, c'est-à-dire les réseaux d'influence. Nous avons ainsi représenté la dimension relationnelle dans notre recherche par le réseau d'influence et le réseau de confiance. Ces aspects relationnels doivent influencer positivement l'usage des TIC.

En synthèse, nous retenons les dimensions du capital social dans ses aspects structurels, relationnels et cognitifs d'une part, pour expliquer l'usage des $\mathrm{TI}$ en mesurant la centralité des utilisateurs de ces $\mathrm{Tl}$ au sein d'un réseau social. C'est ce que décrit notre modèle de recherche ci-après.

\section{Le modèle de recherche}

Parmi les outils des Technologies de l'Information et de la Communication, certains présentent la caractéristique de pouvoir supporter les relations sociales. Nous pensons notamment à des outils répandus tels que la messagerie électronique (Cucchi, 2004), le téléphone mobile, les outils de discussion en ligne tels que Messenger ou Skype, ou encore les outils de réseautage tels que Facebook. En effet, ils ont tous la particularité de mettre en relation un utilisateur avec les autres membres du groupe. 


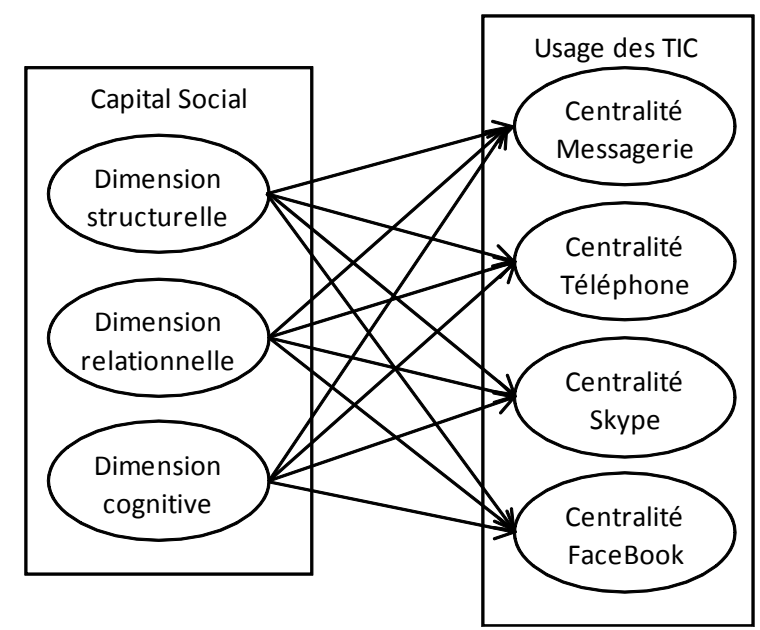

Figure 1 : Le modèle de recherche

Le modèle de recherche (voir figure ci-dessus) vise à montrer que le capital social a une influence sur les usages des différents outils de communication électroniques. Les relations sont testées sur la base des indicateurs de l'analyse des réseaux sociaux. Etant donné qu'il s'agit d'une recherche à caractère exploratoire ; nous ne savons pas a priori les liens significatifs. Nous pouvons donc formuler les hypothèses de recherche suivantes :

$\mathrm{H} 1$ : Il existe une influence significativement positive entre les aspects structurels du capital social et l'usage de la messagerie.

$\mathrm{H} 2$ : Il existe une influence significativement positive entre les aspects relationnels du capital social et l'usage de la messagerie.

$\mathrm{H} 3$ : II existe une influence significativement positive entre les aspects cognitifs du capital social et l'usage de la messagerie.

$\mathrm{H} 4$ : Il existe une influence significativement positive entre les aspects structurels du capital social et l'usage du téléphone.

H5 : Il existe une influence significativement positive entre les aspects relationnels du capital social et l'usage du téléphone.

H6 : Il existe une influence significativement positive entre les aspects cognitifs du capital social et l'usage du téléphone.

$\mathrm{H} 7$ : Il existe une influence significativement positive entre les aspects structurels du capital social et l'usage de Skype.

H8 : Il existe une influence significativement positive entre les aspects relationnels du capital social et l'usage de Skype.

H9 : Il existe une influence significativement positive entre les aspects cognitifs du capital social et l'usage de Skype.

H10 : Il existe une influence significativement positive entre les aspects structurels du capital social et l'usage de Facebook. 
$\mathrm{H} 11$ : Il existe une influence significativement positive entre les aspects relationnels du capital social et l'usage de Facebook.

$\mathrm{H} 12$ : Il existe une influence significativement positive entre les aspects cognitifs du capital social et l'usage de Facebook.

Ce modèle sera testé selon une méthodologie décrite ci-après.

\section{Méthodologie}

\subsection{Le questionnaire}

Les données utilisées pour cette recherche se focalisent sur la dimension collective. Il s'agit de collecter des informations pour rendre compte des dimensions relationnelles. Dans ce but des questions spécifiques ont été recueillies. Nous nous appuyons en l'adaptant à notre sujet d'étude, sur les méthodes de l'analyse des réseaux sociaux (Wasserman, Faust, 1994 ; Lazega, 1998). Les données collectées doivent permettre de rendre compte de la position des individus dans le réseau selon plusieurs dimensions. Ces données prennent la forme d'une liste de noms et d'une mesure indiquant la force de la relation. Par exemple, pour la variable structurale « relation de travail » la question suivante a été posée aux acteurs du groupe :

« Parmi les étudiants de votre promotion, citez les personnes (10 au maximum) avec lesquelles vous aimez travailler (en dehors des cours) ?»

En respectant un masque de saisie, le répondant a alors dû fournir une liste de noms en lui associant la durée hebdomadaire moyenne de travail.

En opérant ainsi, le répondant désigne la liste des acteurs du groupe lui paraissant le plus proche (ou le plus éloigné) en fonction de la variable structurale choisie. Voici la liste des variables structurales utilisées :

- La variable structurale « préférence au travail » pour identifier la configuration générale des relations dans le groupe de travail.

- La variable structurale " capacité à influencer le comportement " pour caractériser les relations de pouvoir au sein du groupe. Ces relations de pouvoir s'entendent au sens large du terme. Pour reprendre la dichotomie opérée par Weber, le pouvoir s'exprimera plutôt ici en termes d'autorité, c'està-dire dans la capacité à faire réaliser volontairement une action par un autre acteur. En effet, entre étudiants il n'existe pas de relations hiérarchiques (ou de nature similaire) susceptibles d'imposer des décisions et des ordres.

- La variable structurale « partage d'une vision » pour caractériser l'homogénéité des représentations des individus dans le réseau. Dans le cas de ce travail, la vision partagée porte sur le comportement des individus au travail, en 
interrogeant sur ce qu'ils considèrent être « le bon comportement individuel en groupe ".

- La variable structurale " confiance » caractérisant la dimension relationnelle entre les acteurs au sein du réseau.

- Les variables structurales relatives aux usages de la technologie : fréquence des échanges de messages, fréquence des échanges téléphoniques, fréquence des échanges par des outils de type Skype/MSN.

L'utilisation de ces variables structurales a pour but de caractériser les relations entre les individus, tant dans la dimension réelle et affective que dans la dimension « virtuelle» des usages de la technologie.

\section{2. Échantillon}

A ce stade de la recherche, l'équipe a conçu un questionnaire. Celui-ci, élaboré avec le logiciel Sphinx, a été mis en ligne auprès des étudiants de l'IAE. Les étudiants sont réunis lors d'une séance de travail et doivent se connecter sur un serveur. La personne en charge de l'administration du questionnaire insiste sur la nécessité de collecter une part significative du groupe. En effet, l'unité d'analyse étant la place de l'individu dans le groupe, un nombre insuffisant de réponses ne permettrait pas de traiter la dimension collective. Les données de 199 étudiants de niveau L3, Master 1 ère et $2^{\text {ème }}$ année appartenant à 11 promotions différentes ont été collectées. Cette première phase a permis de valider le questionnaire et son administration en ligne. II s'agissait notamment de vérifier la méthode de collecte des données relationnelles par questionnaire en ligne. Le modèle de recherche a été traité avec une méthodologie de type "Partial Least Square " (PLS). Elle a été utilisée pour vérifier l'adéquation des données et du modèle de recherche.

\subsection{Les mesures de centralité des acteurs}

En raison du nombre de traitements à effectuer, l'environnement programmable de traitement statistique $R(V$ 2.10.1) a été utilisé. Les packages statnet et igraph ont été utilisés pour automatiser le calcul d'un ensemble d'indicateurs de centralité (voir Tableau 1 pour la liste des indicateurs). Ces derniers sont calculés à partir de plusieurs matrices d'adjacence caractérisant des relations de différentes natures : relations de travail, relations de confiance, proximités des représentations (comportement individuel en groupe), relations d'influence, usage de la messagerie, usage du téléphone, usage de visioconférence de type Skype/MSN, usage de Facebook. Afin de mieux identifier les contextes et leurs influences sur les usages des technologies de l'information, les matrices relatives aux relations de travail et celles relatives aux TIC (messagerie, téléphone, Skype et Facebook) ont été traitées. Les différents indicateurs ont fait l'objet d'une procédure de factorisation afin de dégager les dimensions les plus importantes. 


\subsection{Procédure de factorisation}

Les données utilisées proviennent des calculs de centralité des acteurs. Dans un premier temps, afin d'éviter les facteurs d'échelle et de limiter l'effet des différences de distribution, les données vont être centrées et réduites. La méthode "Listwise deletion " permet de traiter les données manquantes. Le test de sphéricité de Bartlett et le test d'adéquation des données (MSA Measure of Sampling Adequacy) de Kaiser, Meyer et Olkin ont été mis en œuvre pour savoir si les données sont factorisables (Evrard et al., 1997, chapitre 10). Pour construire les facteurs, la méthode du maximum de vraisemblance est utilisée car elle est la plus robuste. Le test de Kaïser (valeur propre supérieure à 1) sera utilisé pour déterminer le nombre de facteurs à retenir. L'alpha de Cronbach sera calculé afin de vérifier la validité du facteur. La valeur de cet indicateur devra être supérieur à 0,70 . Au terme de cette procédure de factorisation, trois facteurs ont été obtenus :

- "Centrality » : Degree, Eigenvector, Hub and Authority

- "Closeness » : Closeness, Closeness in, Closeness Out

- «Betwenness » : Betwenness

\subsection{Traitement du modèle structurel}

Le modèle structurel proposé a été traité avec un mode de traitement de type Partial Least Square (logiciel Smart PLS V2.0M3). II s'agit d'une technique alternative aux méthodes de traitement qui se basent sur l'analyse de covariance. Les raisons associées à ce choix sont de plusieurs ordres. Tout d'abord, ce travail présente un caractère exploratoire. Les méthodes qui se fondent sur l'analyse des structures de covariances (type LISREL) sont adaptées à des recherches à caractère confirmatoire. Elles visent à confirmer les paramètres d'une théorie connue. En cherchant à maximiser la variance expliquée, PLS est plutôt orienté sur la prédiction. De plus, comme souvent dans le domaine des sciences sociales, les données collectées sont entachées de bruit et leurs distributions sont mal ajustées. Ainsi, la condition de normalité des données n'est pas totalement respectée. De ce point de vue, les méthodes PLS sont moins sensibles et acceptent des données dont la distribution est moins ajustée. La taille de l'échantillon et le nombre d'indicateurs nécessaires à la convergence des modèles sont également plus faibles. En synthèse, selon Chin (2000), PLS présente les caractéristiques d'une méthode plus souple, particulièrement adaptée aux recherches à caractère exploratoire.

\section{Résultats}

D'un point de vue méthodologique, le modèle initial a été testé avec les trois mesures de centralité qui se sont dégagées de l'analyse factorielle exploratoire. Dans un but de concision et de clarté dans la présentation des résultats, nous 
avons choisi de nous concentrer sur la première mesure "Centrality ». En effet, les indicateurs associés sont ceux que l'on rencontre le plus fréquemment dans les travaux traitant de l'analyse des réseaux sociaux (notamment les mesures de centralité Degree et Eigenvector). Les résultats présentés testeront le modèle de recherche en utilisant une mesure de centralité composite constituée de Degree, Eigenvector, Hub et Authority. Dans un premier temps, les critères de qualité d'ajustement du modèle vont être examinés. Dans un deuxième temps, les résultats du traitement du modèle structurel vont être discutés.

\subsection{Le modèle final}

Les indicateurs de qualité de l'ajustement du modèle global ont un niveau acceptable. Ainsi, les niveaux de variance extraite (AVE : Average Variance Extracted) sont supérieurs à 0,60 , au-delà de la valeur limite de 0,5 ; les niveaux de fiabilité (CR : Composite Reliability) sont supérieurs à 0,88 et très supérieurs à la limite de 0,7 ; les alpha de Cronbach ( $\alpha$ ) sont supérieurs à 0,83 et montrent la convergence des items vers la variable latente. En termes de validité convergente, Chin (1998) considère que le niveau des AVE doit être supérieur à 0,5. C'est le cas pour l'ensemble des construits. L'évaluation de la validité discriminante vise à s'assurer, dans le cas de mesures réflexives, que la variance partagée par la variable latente et ses items est supérieure à celle partagée avec les autres variables latentes. C'est le cas dans le modèle testé. De plus, les items de chaque mesure chargent plus sur la variable latente qui lui est associée que sur les autres. En conséquence, compte tenu des indicateurs de qualité présentés, nous pouvons considérer que les mesures utilisées satisfont les conditions de validité convergente et de validité discriminante.

Le traitement des données a été opéré en deux temps. Dans un premier temps, les concepts mobilisés dans le cadre de la théorie du « Capital Social » (Confiance et Influence pour les variables relationnelles, Vision pour la dimension cognitive, Centralité au travail pour la dimension structurelle de l'activité étudiée) ont été mis en relation avec les variables relatives aux usages des technologies de l'information et de la communication (centralités dans les réseaux des relations par messagerie, par téléphone, par visio de type Skype/MSN et par Facebook). Une approche par "Bootstrap " a été réalisée de façon à tester les relations sur la base de 300 échantillons aléatoires composés de 100 observations. De cette façon, le traitement de ce modèle initial a permis de révéler les relations structurelles significatives.

Sur la base de ces relations significatives, un deuxième modèle a été construit. II tient compte uniquement des relations structurelles ayant un niveau de signification élevé $(p<0,01)$. 


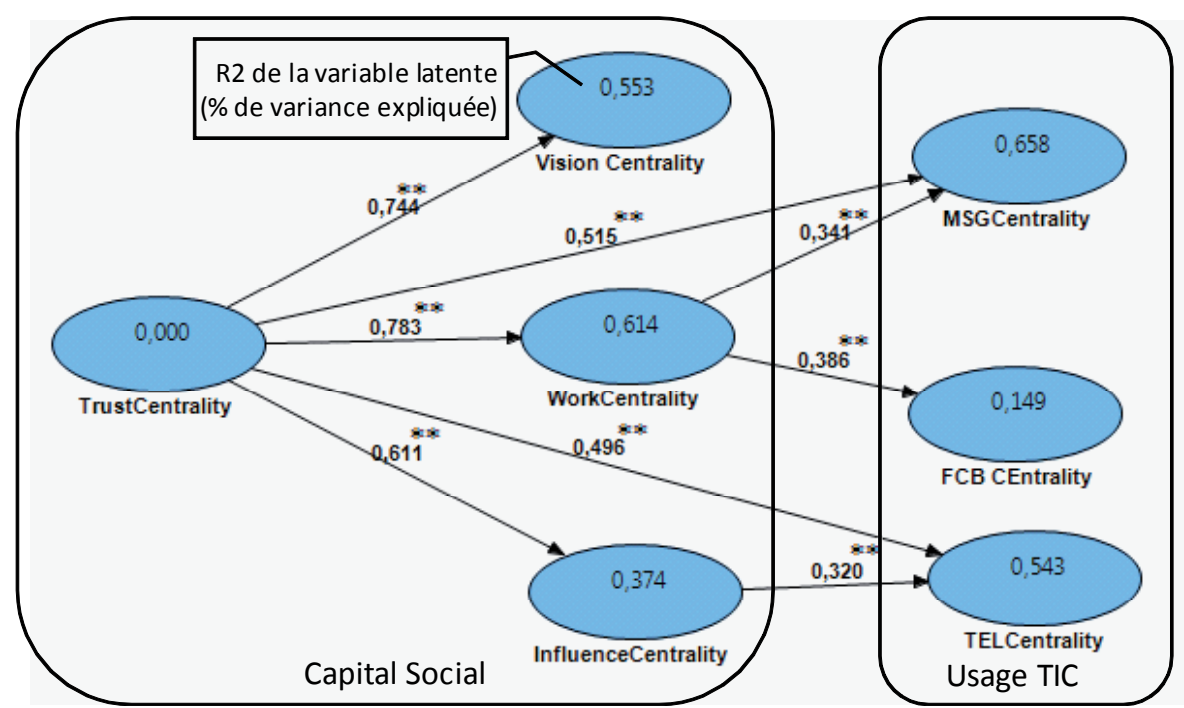

Figure 2 : Le modèle structurel final

Comme nous pouvons l'observer dans la figure ci-avant, deux catégories de concept apparaissent dans le modèle final. Les concepts relevant du capital social en tant que variables explicatives et ceux relevant des usages des technologies en tant que variables expliquées.

Dans les concepts associés au Capital Social, celui associé à la confiance (TrustCentrality) occupe une place particulière. En effet, l'étude des corrélations entre concepts montre que la confiance est corrélée avec les variables de l'usage des TIC (à l'exception de la centralité associée à Skype/MSN) mais également avec les variables du capital social. Considérant la place que cette dimension occupe dans la littérature, nous avons décidé de l'utiliser comme variable permettant d'expliquer les autres variables du capital social : centralité dans le partage de la vision de l'individu au travail, centralité dans le réseau de travail et centralité dans le réseau d'influence.

La lecture de ce schéma de synthèse nous permet de faire un état des lieux des hypothèses en identifiant les relations dont l'effet est significatif.

\begin{tabular}{|c|c|c|c|c|c|}
\hline Hyp & $\begin{array}{c}\text { Intitulé } \\
(\Rightarrow \text { signifie « a un effet sur } »)\end{array}$ & Effet(s) & Hyp & $\begin{array}{c}\text { Intitulé } \\
(\Rightarrow \text { signifie « a un effet su }\end{array}$ & Effet(s) \\
\hline $\mathrm{H} 1$ & $\begin{array}{l}\text { Dim. structurelle } \\
\text { du CS } \Rightarrow \text { usage messagerie }\end{array}$ & Significatif & $\mathrm{H} 7$ & $\begin{array}{l}\text { Dim.structurelle du CS } \\
\Rightarrow \text { usage visio (Skype/MSN) }\end{array}$ & Non significatif \\
\hline $\mathrm{H} 2$ & $\begin{array}{l}\text { Dim.relationnelle } \\
\text { du CS } \Rightarrow \text { usage messagerie }\end{array}$ & $\begin{array}{l}\text { Significatif } \\
\text { confiance } \\
\text { Non significatif } \\
\text { influence }\end{array}$ & $\mathrm{H} 8$ & $\begin{array}{l}\text { Dim.relationnelle du CS } \\
\Rightarrow \text { usage visio (Skype/MSN) }\end{array}$ & $\begin{array}{l}\text { Non significatif } \\
\text { confiance et } \\
\text { influence }\end{array}$ \\
\hline
\end{tabular}




\begin{tabular}{|cll|cl||}
\hline $\mathrm{H} 3$ & $\begin{array}{l}\text { Dim.cognitive } \\
\text { du CS } \Rightarrow \text { usage messagerie }\end{array}$ & Non significatif & $\mathrm{H} 9 \begin{array}{l}\text { Dim.cognitive du CS } \\
\Rightarrow \text { usage visio (Skype/MSN) }\end{array}$ & Non significatif \\
\hline $\mathrm{H} 4 \begin{array}{l}\text { Dim.structurelle } \\
\text { du CS } \Rightarrow \text { usage téléphone }\end{array}$ & Non significatif & $\mathrm{H} 10 \begin{array}{l}\text { Dim.structurelle du CS } \\
\Rightarrow \text { usage Facebook }\end{array}$ & Significatif \\
\hline H5 $\begin{array}{l}\text { Dim.relationnelle du CS } \Rightarrow \\
\text { usage téléphone }\end{array}$ & $\begin{array}{l}\text { Significatif } \\
\text { confiance et } \\
\text { influence }\end{array}$ & $\mathrm{H} 11 \begin{array}{l}\text { Dim.relationnelle du CS } \\
\Rightarrow \text { usage Facebook }\end{array}$ & $\begin{array}{l}\text { Non significatif } \\
\text { confiance et } \\
\text { influence }\end{array}$ \\
\hline H6 $\begin{array}{l}\text { Dim.cognitive du CS } \\
\Rightarrow \text { usage téléphone }\end{array}$ & Non significatif & $\mathrm{H} 12 \begin{array}{l}\text { Dim.cognitive du CS } \\
\Rightarrow \text { usage Facebook }\end{array}$ & Non significatif \\
\hline \hline
\end{tabular}

Tableau 2 : Synthèse des hypothèses

Nous devons noter que certains résultats ne sont pas résumés dans le tableau ci-avant. Ce sont des relations significatives entre les concepts du capital social et relatives au rôle de la confiance.

\begin{tabular}{|ccc|}
\hline Hyp & $\begin{array}{c}\text { Intitulé } \\
(\Rightarrow \text { signifie « a un effet sur })\end{array}$ & Effet(s) \\
\hline H'1 & Dimension relationnelle Confiance $\Rightarrow$ Dimension cognitive Vision & Significatif \\
\hline H'2 & Dimension relationnelle Confiance $\Rightarrow$ Dimension structurelle Travail & Significatif \\
\hline H'3 & Dimension relationnelle Confiance $\Rightarrow$ Dimension relationnelle Influence & Significatif \\
\hline
\end{tabular}

Tableau 3 : Résultats complémentaires

Nous pouvons observer que ces résultats placent la confiance au cœur du capital social. La centralité dans le réseau de confiance est en relation directe avec la centralité de la vision " du bon comportement individuel en groupe », celle de l'individu au travail, celle de l'influence dans le réseau.

\section{Discussion}

Globalement, le capital social, décrit par la centralité dans les réseaux de confiance, d'influence, de partage des visions et la centralité dans les activités de travail, a un effet sur les usages des technologies de l'information et de la communication. Toutefois, les résultats présentés ci-avant appellent un ensemble de commentaires.

Dans un premier temps, la lecture des résultats nous fait observer que les niveaux d'explication des variables dépendantes associées à la technologie diffèrent. En effet, le modèle testé permet d'expliquer près de $66 \%$ de la variance de la centralité dans les réseaux supportés par la messagerie $\left(R^{2}=0,658\right)$, plus de $54 \%$ de la variance de la centralité dans les réseaux supportés par le téléphone $\left(R^{2}=0,543\right)$ et moins de $15 \%$ pour Facebook $\left(R^{2}=0,149\right)$. II faut également noter l'absence de la technologie Skype/MSN, aucun effet statistiquement significatif n'ayant 
pu être identifié. Nous pouvons considérer que, dans le contexte de travail des étudiants, le capital social produit des effets d'intensités différentes selon les TIC. L'intensité la plus importante concerne la messagerie et le téléphone. L'intensité la plus faible concerne Facebook. Nous pouvons interpréter cette situation en référence au contexte d'usage. En effet, le contexte général est celui du travail des étudiants et la façon dont ils s'organisent pour réaliser leurs travaux. La position relative de chacun d'eux dans les réseaux de travail a un effet significatif sur leur position dans les réseaux associés à la messagerie. Le contexte d'usage de ce média est donc lié à celui du travail. Une lecture en creux nous permet de noter l'absence de relations significatives avec Skype/MSN. C'est un usage qui n'est pas associé au contexte de travail des étudiants. En effet, la position des individus dans les réseaux de travail est non significativement corrélée à celle occupée dans les réseaux Skype/MSN. Une interprétation possible est la concurrence de Facebook qui dispose de fonctionnalités plus appropriées à leur usage. En effet, la visioconférence nécessite un ordinateur avec caméra et un abonnement avec un débit minimal pour pouvoir fonctionner. De plus, les étudiants se rencontrent souvent et n'ont donc pas forcément besoin de visualiser leurs interlocuteurs.

En revanche, la centralité dans le travail affecte significativement l'usage de Facebook (coeff. 0,386). Le niveau de R2 étant faible, cela signifie que d'autres aspects interviennent dans la centralité associée à ce média. Nous pouvons supposer que les relations établies dans le cadre des activités de travail se prolongent sur Facebook. Toutefois, d'autres réseaux sociaux viennent probablement se superposer à celui des relations de travail. Les caractéristiques d'usage de cet outil reflètent donc partiellement les positions dans les relations de travail. Considérant l'engouement pour ces technologies, on peut raisonnablement supposer que ces technologies relèvent plutôt d'un contexte d'usage plus ludique et de loisir. Pour vérifier cette interprétation, des questions complémentaires devraient être posées afin d'identifier la structure du réseau associée à des relations de loisir.

En examinant plus attentivement les éléments du capital social qui ont un effet significatif sur la messagerie et le téléphone, on observe ici aussi des caractéristiques propres à chaque média. La centralité dans la messagerie est directement sous l'effet de la centralité au travail (coeff. 0,341) et de la confiance (coeff. 0,515 ). Son usage est donc significativement lié à la structure des relations de travail et de confiance dans le groupe. Les variables ayant un effet significatif sur la centralité dans l'usage du téléphone sont partiellement différentes. Ici encore, la confiance a un effet direct (coeff. 0,496). La confiance affecte donc significativement la centralité dans l'usage du téléphone. En revanche, la centralité au travail n'a pas d'effet significatif sur l'usage du téléphone. L'autre variable ayant un effet significatif est la centralité dans les réseaux d'influence. Plus un individu est central dans le réseau d'influence, plus il sera central dans l'usage du 
téléphone. Rappelons que l'influence caractérise les individus susceptibles de faire changer d'avis, de modifier les comportements. L'interprétation de ces résultats doit tenir compte également de la nature particulière du téléphone. En effet, cette technologie est banale actuellement et presque la totalité des étudiants y ont accès. Toutefois, elle diffère par la nécessité de payer la transaction téléphonique. En effet, la mise à disposition de l'Internet auprès des étudiants leur permet de communiquer facilement et sans frais supplémentaires par messagerie. Ce n'est pas le cas pour le téléphone où le coût de la communication n'est pas négligeable, notamment dans le cas des étudiants. Cela se traduit notamment par une densité des réseaux associés à ce média bien plus faible que pour la messagerie. Ainsi, la communication par téléphone a un coût et la décision de communiquer par ce média est généralement plus réfléchie que dans les autres cas : c'est une action plus engageante financièrement parlant. Dans cette situation, l'utilisateur va réserver son usage aux personnes importantes à ses yeux, c'est-à-dire celles dont l'avis compte et qui sont susceptibles de l'influencer. La centralité dans la dimension influence a alors un effet significatif (coeff. 0,320) sur la centralité dans l'usage du téléphone. Ceci laisse supposer que l'usage du téléphone est plus centré sur la sphère intime, en relation avec les personnes dont l'avis peut influencer le comportement de l'étudiant.

En ce qui concerne la variable « vision », elle traduit la centralité des acteurs dans le réseau des représentations du « bon comportement individuel en groupe ». La centralité dans ce réseau est significativement affectée par la centralité dans le réseau de confiance (coeff. 0,744). Toutefois, cette variable n'a pas d'effet significatif sur les usages des TIC étudiées. La dimension cognitive du capital social, telle qu'elle a été appréhendée, n'a donc pas d'effet particulier sur les usages des TIC. II serait toutefois prématuré d'en conclure une absence définitive d'effet. En effet, les promotions considérées partagent une formation sur 2 à 3 années, avec des départs et des nouveaux arrivants chaque année. Ce contexte est donc moins propice au développement de la dimension cognitive (culture et connaissances partagées notamment). Des recherches complémentaires devraient permettre de mieux caractériser cette dimension.

Enfin, en ce qui concerne la confiance, on peut observer l'importance de ce concept pour comprendre la dynamique liée au capital social. Cette variable latente a un effet significatif sur l'ensemble des autres variables du capital social d'une part, et sur l'usage de la messagerie et du téléphone d'autre part. Toutefois, elle n'affecte pas de façon significative les usages des médias Skype/MSN et Facebook. L'usage de ces médias peut donc se développer indépendamment de la position des individus dans les relations de confiance. Cela suppose donc que la nature des relations sur ces supports est moins impliquante. En effet, la confiance est un élément nécessaire à la régulation des relations lorsqu'il y a un risque de comportement opportuniste. Les approches contractualisantes telles que celles inspirées par la théorie des coûts de transaction sont difficilement 
envisageables dans le contexte des relations de travail. Dans le contexte des relations de travail d'étude, le risque d'opportunisme est celui du passager clandestin qui profite des apports du groupe sans proposer d'autres ressources en échange. Cela peut également concerner des relations faisant intervenir de l'affect, où la confiance sert à rassurer l'autre sur son bon comportement. Sur Facebook et Skype/MSN, les relations entretenues ne nécessitent pas ou moins de confiance. Si le risque de comportement opportuniste est faible, c'est que les enjeux de la relation le sont également. Actuellement et pour les étudiants de l'échantillon, ces médias entretiennent probablement des relations sans enjeux, probablement ludiques et/ou de loisir, trouvant leurs sources dans les relations de travail (Exemple : copains de promotions).

\section{Conclusion}

Cet article développe des hypothèses relatives à une recherche en cours. Dans une première partie théorique, les principales théories liées à l'usage des technologies de l'information sont décrites. L'accent est mis sur la difficulté, par ces approches, à rendre compte de la dimension sociale en général et des aspects relationnels en particulier. Nous proposons de nous appuyer sur le capital social et sur l'analyse des réseaux sociaux pour mieux intégrer la dimension relationnelle dans l'usage des TI. Des hypothèses de recherche sont alors exprimées. Dans une deuxième partie, des informations relatives à la méthodologie sont explicitées. Elles développent notamment le recueil des données, les indicateurs utilisés, la méthode de traitement de type PLS et les critères d'ajustement utilisés. Enfin, dans une troisième partie, une discussion des résultats est développée.

Le principal intérêt pour la recherche est d'intégrer la place des individus dans un réseau en tant que mesure. L'approche classique à base de questionnaire et d'échelle de perception prend pour unique référence le point de vue de l'interrogé. Ce sont en général des échelles de Likert permettant de mesurer la perception de l'interrogé. Ce faisant, la dimension collective ne peut être appréhendée qu'au travers des perceptions individuelles vis-à-vis d'un collectif globalisé. Exemple de question : Avez-vous confiance dans les autres membres du groupe?

L'approche que nous proposons utilise les indicateurs de centralité de l'analyse des réseaux sociaux pour capter l'influence du collectif. En permettant de positionner le répondant dans un réseau de relations, ces indicateurs permettent d'évaluer son « importance » dans le collectif en tenant compte de la nature de la relation. Par exemple, en considérant la relation de confiance, la question devient : En qui avez-vous confiance ? Les indicateurs de centralité permettent d'obtenir des mesures relatives à la position des acteurs dans le réseau de confiance. 
Ainsi, l'usage de l'analyse des réseaux sociaux a permis de révéler l'importance de la position des acteurs dans les réseaux des relations. En tant qu'acteur dans un système sociotechnique, l'individu occupe une position qui affecte son comportement en général et son usage des TIC en particulier. Les résultats des travaux permettent de mieux articuler la nature des ressources du capital social dans un contexte de travail et l'usage des technologies de l'information et de la communication. Les TIC (messagerie, téléphone, Skype/MSN et Facebook) présentent différents contextes d'usage. L'usage de la messagerie est associé à la centralité au travail et à la centralité dans la confiance. Celui de Facebook est associé à la centralité au travail. Ces deux médias sont en conséquence sensibles au contexte de travail. En revanche, l'usage du téléphone est associé à la centralité dans le réseau d'influence et dans celui de la confiance. Considérant de plus le caractère payant des transactions, ce média semble plus associé aux relations de la sphère intime, destiné essentiellement aux personnes importantes. Enfin, les outils de visiophonie de type Skype/MSN n'apparaissent pas de façon significative.

D'un point de vue managérial, ces résultats montrent l'influence des relations sociales dans le fonctionnement des organisations. Ces relations, constituant la dynamique du capital social, affectent directement et indirectement l'usage des technologies de l'information et de la communication. Les managers et responsables d'entreprises perçoivent mieux les liens unissant le contexte social et l'usage des technologies ne revêtant pas ou peu un caractère obligatoire. Or ces technologies sont utilisées intensivement par les organisations devant supporter les processus collaboratifs de production. Ce sera notamment le cas des équipes projets situées dans des lieux différents, où l'usage des TIC est primordial.

Au-delà des résultats bruts, cette approche permet potentiellement de faciliter les décisions de gestion. En termes de diagnostic, cette approche par l'analyse des réseaux sociaux propose également de fournir des cartes de relations entre les individus. Ces cartes sont révélatrices des fonctionnements et des dysfonctionnements collectifs (clans, personnes/services isolés, centralisation des échanges...). Elles permettent aux acteurs d'interpréter des situations de gestion. En termes de résultats, cette approche permet de rendre compte des effets de certaines décisions de gestion. Nombre de décisions managériales modifient la carte des relations entre salariés et/ou sous-traitants : recrutement, réorganisation des services, promotion des individus, formations, réunions, séances de motivation, réunions festives... sont autant de décisions pouvant modifier la position des acteurs. Les outils mis à disposition permettent de mieux appréhender la nature des effets associés à ces décisions et d'en évaluer l'intérêt. 
Cette recherche à caractère exploratoire a été réalisée à partir de réponses d'étudiants. Si cet échantillon permet d'évaluer la pertinence de certaines hypothèses, elle présente un caractère spécifique dont il faut tenir compte. A titre d'illustration, la nature de la population étudiée influence probablement l'interprétation liée à l'usage du téléphone. Dans le cas de l'usage du téléphone en entreprise, les terminaux peuvent être pris en charge par l'organisation. C'est le cas par exemple des commerciaux, des cadres de direction, des personnels itinérants/mobiles comme les transporteurs... La contrainte financière étant différente, les usages devraient probablement en être affecté.

Différentes pistes s'ouvrent à ce stade de la recherche. Nous nous centrerons sur deux aspects.

Sur la nature de l'échantillon, nous souhaitons élargir le travail à des contextes professionnels : équipes de développement, personnels de santé... En effet, si les promotions des étudiants permettent de rendre compte de dynamiques de groupe au travail, ils ne peuvent rendre compte des contraintes professionnelles. Outre les limites liées à l'usage du téléphone mobile, nous devons également constater l'absence de hiérarchie imposée dans le groupe. Dans les organisations classiques, une hiérarchie officielle régule les décisions et le pouvoir des acteurs. Rien de tel dans une promotion d'étudiants. Toutefois, les organisations informelles et collaboratives se développent. A titre d'exemple, mais pas uniquement, nous pouvons citer la production logicielle Open-Source, les productions collaboratives structurées autour de Wiki... Les démarches telles que celles proposées dans ce travail peuvent fournir des pistes pour faciliter le management de telles organisations.

Sur les facteurs affectant la position des acteurs, nous ne pouvons avancer des interprétations à ce stade du travail. En effet, la méthode permet de constater une position, mais elle ne permet pas de l'expliquer au sens de l'identification de causes affectant la position des acteurs. Or cette connaissance serait très utile pour la compréhension des dynamiques en œuvre et potentiellement remédier à des dysfonctionnements (acteurs isolés, groupes fragmentés, clans...). C'est un axe qui sera au cœur des développements futurs de la recherche.

\section{Références}

Ajzen I. (1991), «The theory of planned behavior», Organizational Behavior and Human Decision Processes, 50(2), pp. 179-211.

Bergeron F., Raymond L., Rivard S. et Gara M.F. (1995), "Determinants of EIS Use: Testing a Behavioural Model", Decision Support Systems, n 14, 1995, pp. 131-146.

Bonacich P. (1987), "Power and Centrality: A Family of Measures", American Journal of Sociology, 92(5), pp. 1170-1182.

Boukef N. et Kalika M. (2000), « Facteurs déterminants de l'utilisation du courrier électronique ", 5 ${ }^{\text {ème }}$ congrès de l'AIM, 2000. 
Bourdieu P. (1980), "Le capital social. Notes provisoires », Actes de la recherche en sciences sociales, vol. 31, janvier, pp. 2-3

Bourdieu P. (1985), "The Forms of Capital," in Handbook of Theory and Research for the Sociology of Education, J. G. Richardson (Ed.), Greenwood Press, New York

Brass D. J. et Burkart M.E., (1992), "Centrality and power in organisations", Chapter 7 in Nohria and Eccles (Eds) Networks and Organisations: Structure Form and Action, Harvard Business School Press, Boston Mass pp.191-215.

Burt R. (1982), Toward a Structural Theory of Action, Network Models of Social Structure, Perception, and Action, Academic Press, New York, 381p.

Burt R. (1980), "Models of Network Structure", Annual Review of Sociology, 6, pp. 79141.

Burt R. (1992), "The Social Structure of Competition", in Nohria and Eccles (Eds) Networks and Organisations: Structure Form and Action, Harvard Business School Press, Boston Mass, Chapter 2.

Chin Wynne W. (2000), "Partial Least Squares for Researchers: An overview and presentation of recent advances using the PLS approach", http://disc-nt.cba.uh.edu/chin/ icis2000plstalk.pdf

Chiu C-M., Hsu M-H et Wang, E.T.G (2006), "Understanding knowledge sharing in virtual communities: An integration of social capital and cognitive theories", Decision Support Systems, 42 (2006), pp. 1872-1888

Coleman JS (1988), « Social Capital in Creation of Human Capital », The American Journal of Sociology, Vol. 94, Supplement S95-S120

Contractor N.S. et Eisenberg E.M. (1990), "Communication networks and new media in organizations". In J. Fulk \& C.W. Steinfield (Eds.), Organizations and communication technology: 143-172, Newbury Park, CA : Sage.

Cucchi A., Fuhrer C. et Picard P. (2009), " Les capacités relationnelles des technologies de l'information : le concept de capital technico-social a-t-il un sens " ? 14 ${ }^{\text {ème }}$ colloque de I'AIM, 2009, Marrakech.

Davis F. D. (1989), "Perceived usefulness, perceived ease of use, and user acceptance of information technology”, MIS Quarterly, 13(3), pp. 319-339.

Degenne A. et Forsé M (1994), Les réseaux sociaux, Armand Colin, 288p.

Desanctis G. et Poole M.S. (1994), "Capturing the complexity in advanced technology use: Adaptive structuration theory", Organization Science, 5(2), May 1994, pp 121-146.

Evrard P., Roux C. et Dussaix, C. (1997), Market, Etudes et recherches en marketing, 2ème édition, Ed. Nathan, Collection Connaître et pratiquer la gestion, 672 p., Paris.

Fishbein M. et Ajzen I. (1975), Belief, attitude, intention, and behavior: An introduction to theory and research. Reading, Mass.; Don Mills, Ontario: Addison-Wesley Pub. Co

Freeman L.C. (1979), "Centrality in Social Networks; Conceptual Clarification", Social Networks, 1(1978/79), pp. 215-239.

Giddens A. (1988), La constitution de la société, PUF, Paris, 1988, 474p.

Granovetter M. S. (1973), "The Strength of Weak Ties", American Journal of Sociology, 78 (6), pp. 1360-1380.

Josserand E. (2001), L'entreprise en réseau, Vuibert, Entreprendre, 126p

Kalika M. (2000), « Le management est mort, vive le e-management! », Revue Française de Gestion, FNEGE, juin-juillet-août 2000, pp 68-74.

Kalika M. (2002), Les défis du management, 15 réflexions pour l'action managériale dans un environnement turbulent, Editions Liaisons, 2002, 238p

Kalika M., Boukef N. et Isaac H. (2005), « La théorie du millefeuille. De la non substitution entre communications électronique et face à face ", Communication du $10^{\text {ème }}$ congrès de l'AIM, Toulouse, 2005.

Lamb R. et Kling R. (2003), «Reconceptualizing users as social actors in information systems research», MIS Quarterly, 27(2), pp. 197-235. 
Laumann E. et Pappi F. (1976), Networks of Collective Action; a Perspective on Community Influence System, New York Academic Press.

Laumann E.O., Galskeiwicz L. et Marsden P.V. (1978), "Community Structure as Interorganizational Linkages“. Annual Review of Sociology, Vol 4, pp. 455-484.

Lazega E. (1998), Réseaux sociaux et structures relationnelles, Presses Universitaires de France, Collection «Que sais-je ? ", $127 \mathrm{p}$.

Limayem M., Bergeron F. et Richard A. (1997), « Utilisation des messageries électroniques : mesures objectives versus mesures subjectives ", Revue Systèmes d'Information et Management, 1 (2), pp 51-69.

Martti S. (2000), "Two Concepts of Social Capital: Bourdieu vs. Putnam", ISTR Fourth International Conference, Trinity College, Dublin, Ireland, July 5-8, 2000

Montes JC. et Pronovost S. (2007), "La conversation sur les notions de capital social et de réseau : à la recherche d'une "canne blanche " ", XVlème Conférence de l'AIMS.

Moreno J-L. (1970), Les fondements de la sociométrie (Who shall survive ?), PUF, 2ème édition, 1970, 501p

Nahapiet J. et Ghoshal S. (1997), "Social Capital, Intellectual capital and the creation of value in firms", Academy of Management Proceedings.

Nahapiet J. et Ghoshal S. (1998), "Social Capital, Intellectual Capital, and the Organizational Advantage", Academy of Management Review, 23(2), pp.242-266.

Nohria N. (1992), Introduction: "Is a network perspective a useful way to studying organisations", in Nohria and Eccles (Eds) Networks and Organisations: Structure Form and Action, Harvard Business Scholl Press, Boston Mass, 22p.

Polanyi M. (1967), The Tacit Dimension, London: Routledge and Kegan Paul, First published in 1966

Putnam Robert D. (2000), Bowling Alone : The Collapse and Revival of American Community. New York: Simon \& Schuster

Putnam Robert D. (2004), L'Observateur de l'OCDE, N²42, Mars 2004, www. observateurocde.org

Putnam Robert D. (1993), Making democracy work. Civic traditions in modern Italy. Princeton: Princeton University Press 1993

Putnam, Robert D. (1995), "Bowling alone: America's declining social capital", Journal of Democracy, 6 (1), pp. 64-78.

Rai A., Maruping L-M. et Venkatesh V. (2009), « Offshore Information Systems Project Success: The Role of Social Embeddedness and Cultural Characteristics", MIS Quarterly, 33 (3), pp.617-641.

Rogers E-M. (1995), Diffusion of Innovations. 4thed. New York: Free Press.

Rowe F. et Beal L. (1998), « Les freins structurels et culturels à l'usage de la messagerie électronique dans la banque », L'harmatton, Gresico-Vannes, 10 et 11 septembre 1998.

Rowe F. (2002), "Communication et coopération à distance ", Extrait de Faire de la Recherche en Systèmes d'Information, coordonné par Frantz Rowe, Vuibert, FNEGE, pp. 173-200, 359p., 2002.

Short J, Williams E. et Christie B., (1976), The Social Psychology of Telecommunications, John Wiley and Sons, New York, 1976, 195p.

Tichy N. M. (1981), Networks in organizations, in Nystrom and Starbuck (Eds) Handbook of Organizational Design, Vol. 2, Oxford University Press, New York, 225-249, 552p.

Venkatesh V., Morris M-G., Davis G.B. et Davis F.D. (2003); "User acceptance of information technology: Toward a unified view”, MIS Quarterly, 27(3), pp. 425-478.

Wasserman S, et Faust K. (1994), Social Network Analysis: Methods and Applications, Cambridge University Press, Cambridge, England, 825p.

Watzlawick P., Helmick B. et Jackson D. (1967), "Pragmatics of Human Communication. A study of Interactional Patterns, Pathologies, and Paradoxes". W.W. Norton \& Compagny, inc. New York, 1967. Traduction française par MORCHE J. : Une logique de la communication, 
Paris, Editions du Seuil, 1972, 280p.

Yang S., Lee H. et Kurnia, S. (2009), "Social Capital in Information and Communications Technology Research: Past, Present, and Future", Communications of the Association for Information Systems, Volume 25, Article 23.

Yates J. Orlikowsky. (1992), "Genres of organizational communication: a structurational approach to studying communication and media", Academy of Management Review, 17 (2), pp. 299-326.

Yates J. et Wanda O. (2002), "Genre Systems: Structuring Interaction through Communicative Norms.", Journal of Business Communication, 39 (1), pp. 13-35. 